\title{
Internacionalização do Ensino, da Pesquisa e Inovação: Estudo de caso da Universidade de Brasília (UnB)
}

\author{
Internationalization of Education, Research and Innovation: a case \\ study of the University of Brasilia (UnB)
}

\author{
Regina Coeli Andrade Marques ${ }^{1}$ \\ Maria Hosana Conceição ${ }^{1}$ \\ ${ }^{1}$ Universidade de Brasília, Brasília, DF, Brasil
}

\begin{abstract}
Resumo
Este estudo descreve e analisa o processo de internacionalização da Universidade de Brasília. A metodologia foi a pesquisa bibliográfica e documental na base de dados da Assessoria de Assuntos Internacionais INT da Universidade de Brasília (UnB). As ações de internacionalização se iniciam nas Unidades Acadêmicas, pelos professores, com a realização de acordos de cooperação científicos e tecnológicos com diversas IES exteriores, acordos que a Assessoria de Assuntos Internacionais (INT) negocia promovendo a mobilidade de docentes/pesquisadores e discentes. Os programas de intercâmbio executados pela INT recebem os alunos internacionais, mas, por outro lado, também enviam os alunos da UnB ao exterior. A INT disponibiliza modelos de acordos em quatro línguas e realiza as etapas para acordos de cooperação acadêmica. Verificou-se que a localização da UnB, próxima aos centros decisórios nacionais, das embaixadas, dos organismos internacionais, das agências de fomento e de outros parceiros, é um diferencial à sua internacionalização, a qual, ao divulgar o conhecimento adquirido, realiza sua inovação acadêmica continuamente.
\end{abstract}

Palavras-chave: Internacionalização do Ensino. Pesquisa. Inovação.

\begin{abstract}
This study describes and analyzes the internationalization process of the University of Brasilia. The methodology was bibliographic and documentary research in the database of the International Affairs Advisory INT of the University of Brasilia (UnB). The internationalization actions start in the Academic Units, by the teachers, with the accomplishment of scientific and technological cooperation agreements with several external IESs, agreements that the Advisory of International Affairs (INT) negotiates promoting the mobility of professors/researchers and students. The exchange programs carried out by INT receive international students but, on the other hand, they also send UnB students abroad. INT provides agreement models in four languages and performs the steps for academic cooperation agreements. It was found that the location of UnB, close to national decision-making centers, embassies, international organizations, development agencies and other partners, is a differential to its internationalization, which, by disseminating the acquired knowledge, makes its innovation academic continuously.
\end{abstract}

Keywords: Internationalization of Education. Search. Innovation.

Área Tecnológica: Políticas Públicas. Internacionalização Acadêmica. 


\section{Introdução}

A economia globalizada exige das universidades, tanto no Brasil quanto no mundo, o preparo de seus estudantes para atuarem como profissionais com perfil adequado às necessidades do mercado de trabalho local e internacional, tanto por meio de currículos mais "internacionalizados", baseados em boas práticas de mobilidade acadêmica, quanto por um direcionamento institucional coerente e adequado às especificidades de cada instituição e ao perfil dos estudantes.

Esse estudo busca responder a questão do significado da internacionalização do ensino, da pesquisa e da inovação e extensão de uma instituição de Ensino Superior no mundo e no Brasil e, porque e como, atualmente, se realiza a internacionalização da Universidade de Brasília (UnB), ou melhor, pretende-se verificar se a proposta da internacionalização da UnB é reforçar as atividades de ensino, pesquisa, inovação e extensão ao buscar a melhoria na formação dos seus estudantes, bem como a integração internacional das atividades de pesquisa, de reflexão e de publicação de trabalhos científicos de seus professores e pesquisadores.

Trata-se de um estudo de caso que analisa o processo de internacionalização da UnB, que atualmente conta com um Plano de Internacionalização a ser desenvolvido no período de 2018 a 2022. Esse plano foi definido por sua comunidade acadêmica e está em fase de acelerada implantação pela Assessoria de Assuntos Internacionais (UnB, 2018b).

Uma Universidade criada logo após a inauguração de sua cidade sede, Brasília, no Distrito Federal, em 21 de abril de 1962, com imensos desafios, em uma época de grandes modificações no cenário nacional, exigiu a crença de muitos em um novo ideal de educação para uma jovem democracia. Estes vieram de todas as regiões do Brasil, e de inúmeros países para montar uma estrutura física e acadêmica. Assim começou a internacionalização da UnB - uma internacionalização em casa e internacional, ao mesmo tempo, para erguer uma instituição com vocação para a inovação, transnacional, multicultural, multiliguista, diversificada e acolhedora desde a época de sua criação (MORHY, 2003).

\section{Referencial Teórico}

A compreensão do estado da arte da internacionalização na Universidade de Brasília está associada, também, à criação da cidade de Brasília, que, sendo um centro de decisões do Governo Brasileiro, promove os trabalhos de cooperação entre o Brasil, representado pela UnB e outros países. A criação da INT culminou com a evolução da vida acadêmica da UnB, ao longo de três décadas e exigiu a mobilidade tanto de estudantes quanto de docentes. Nos itens a seguir, estão descritos os principais conceitos que caracterizam a internacionalização na UnB.

\subsection{O Conceito de Internacionalização da Educação Superior}

O que caracteriza uma Universidade Internacional? O conceito de Internacionalização da Educação Superior é muito complexo e recebe forte influência cultural, social e política. Segundo Knight (2003), na década de 1990, havia um debate para considerar se a educação 
superior internacional seria uma educação comparada, uma educação global ou uma educação multicultural. Mas esse debate evoluiu para outro conjunto de termos: educação transnacional, educação sem fronteiras e educação transfronteiriça.

Knight (2003) destaca que o desenvolvimento da educação a distância impactou consideravelmente todos esses termos, pois as fronteiras geográficas se dissolveram $e$ o foco voltou-se para a responsabilidade, a credibilidade e a garantia de financiamento para a disseminação do conhecimento nas diversas instituições de educação superior do mundo.

Essa mesma autora define a internacionalização da educação superior nacional, setorial $e$ institucional "[...] como o processo de integração de uma dimensão internacional, intercultural ou global na finalidade, função ou na oferta de educação superior" (KNIGHT, 2003, p. 12).

Em 2015, o pesquisador Hans De Wit (2015, p. 2) propôs uma nova definição para a internacionalização:

O processo intencional de integração de uma dimensão internacional, intercultural ou global ao propósito, funções à educação pós-secundária, para melhorar a qualidade da educação e pesquisa para todos os alunos e funcionários, e para fazer uma contribuição significativa para a sociedade.

As universidades ainda são uma inovação em curso. Quando do descobrimento do Brasil, existiam apenas 62 delas no mundo e, em 1800, esse número era apenas de 143. Na América Latina, a de Santo Domingo surgiu em 1538, depois veio a de Lima e a do México, ambas em 1551, criadas por ordens religiosas. No início do século XX, as universidades de Bolonha, Oxford, Paris já tinham cerca de 700 anos e a Harvard e a Yale existiam há mais de 220 anos (MORHY, 2004).

\subsection{A Criação das Universidades no Brasil}

No Brasil, apenas em 1920 teve início a Universidade do Rio de Janeiro, para a outorga do título de Doutor Honoris Causa ao Rei Alberto I, da Bélgica, que veio ao Brasil. Depois foi denominada Universidade do Brasil e, mais tarde, Universidade Federal do Rio de Janeiro, como resultado da união da Escola Politécnica, da Faculdade de Medicina e da Faculdade de Direito, que então existiam (MORHY, 2003, p. 21).

Em 1934, foi criada a Universidade de São Paulo e, nas décadas de 1930 e 1940, a Federal de Minas Gerais, de Pernambuco, da Bahia e a Católica do Rio de Janeiro, do Paraná e do Rio Grande do Sul. No início da década de 1960, cerca de 20 universidades estavam em funcionamento no País (MORHY, 2004).

Segundo os dados publicados pelo INEP (2018) do Censo da Educação Superior de 2017:

Em 2017, o Brasil tinha 296 Instituições de Educação Superior (IES) públicas e 2.152 privadas, o que representa $87,9 \%$ da rede. Das públicas, $41,9 \%$ são estaduais; $36,8 \%$, federais e $21,3 \%$, municipais. Quase 3/5 das IES federais são universidades e 36,7\% são Institutos Federais de Educação, Ciência e Tecnologia (IFs) e Centros Federais de Educação Tecnológica (Cefets). (INEP, 2018) 


\subsection{A Universidade de Brasília (UnB)}

Em 15 de dezembro de 1961, foi instituída a Fundação Universidade de Brasília com o objetivo de criar e manter a Universidade de Brasília (UnB), fundada em 15 de janeiro de 1962 (quando 426 estudantes foram matriculados). Desde sua criação, essa instituição iniciou seu processo de internacionalização acadêmica quando convidou renomados pesquisadores para compor sua equipe acadêmica. A nova Universidade adotou o sistema Institutos/Faculdades/ Unidades Complementares (MORHY, 2004; UnB, 2011).

O perfil internacional da UnB se acentuou progressivamente com a formação acadêmica internacional da maioria de seus professores/pesquisadores e estudantes, com uma mobilidade acadêmica crescente ao longo dos anos, e com o número cada dia mais elevado de acordos bilaterais para pesquisas e intercâmbio.

A UnB promove a integração internacional por meio de diversos mecanismos de trabalho colaborativo com as suas unidades (Faculdades, Institutos e Centros temáticos), sendo um dos elos em diversas redes universitárias, cátedras de estudos, organização de fóruns de discussão dos mais relevantes temas da atualidade, entre outros, que têm na sua Assessoria de Assuntos Internacionais (INT) o suporte necessário ao desenvolvimento de suas parcerias estratégicas.

\subsection{Um Breve Histórico da INT}

Com a criação do Centro de Apoio a Intercâmbio e Programas Internacionais na UnB, ligado ao Gabinete do Reitor, em 1987, iniciou-se uma sistematização das ações de internacionalização da UnB. Inicialmente, o objetivo era viabilizar programas de mobilidade acadêmica da comunidade universitária para troca de experiências culturais e o aperfeiçoamento em diversos idiomas estrangeiros.

Passados dez anos, o Centro de Apoio a Intercâmbio e Programas Internacionais foi transformado em Assessoria de Assuntos Internacionais (INT), uma denominação mais adequada à política e aos interesses da Universidade para celebra novos convênios e acordos de cooperação técnica, científica e cultural com instituições internacionais.

Assim, na UnB, a INT é o órgão que oferece apoio aos professores e estudantes internacionais da $\mathrm{UnB}$, atua nos programas de mobilidade e como um elo entre a UnB e as organizações internacionais como Unesco, Erasmus Mundus Master Course in Work, Erasmus + Organizational, and Personnel Psychology (WOP-P) e mais de 250 Institutos de Ensino Superior Internacionais (IESI) (UnB, 2017b).

\subsection{Acordos de Cooperação}

A INT é responsável por formalizar acordos de cooperação internacional, de natureza bilateral ou multilateral, acadêmica, técnica e científica da $\mathrm{UnB}$ em todas as áreas de interesse das instituições conveniadas, como iniciativa de negociação do corpo docente com diversas instituições de ensino superior internacionais, seguindo as normas jurídicas do Brasil para acordos acadêmicos. A INT conta com equipe própria para a tramitação dos acordos antes das respectivas assinaturas formais. 
A comunidade docente da UnB propõe acordos com as mais diversas instituições de ensino e pesquisa internacionais por intermédio da INT. Embora cada Instituto ou Faculdade da UnB identifique e defina com quais universidades e organismos internacionais querem estabelecer suas parcerias, a instituição busca mapear e dar uma coerência às iniciativas de internacionalização que partem das unidades e dos pesquisadores.

Entre as modalidades de Acordos, existe a Cotutela ou Co-orientação de Tese de Doutorado e dissertação de Mestrado, modalidade que permite ao doutorando e ao mestrando, regularmente matriculados na UnB ou na instituição convenente, a obtenção do título de doutor ou de mestre em ambas as instituições.

A Faculdade de Tecnologia detém o maior número de acordos internacionais devido à evolução tecnológica e inovação nas últimas décadas. Outras unidades também ampliaram nos últimos anos uma maior internacionalização nos seus Programas de Pós-Graduação e Pesquisa, bem como de inovação.

$\mathrm{Na}$ INT, verifica-se que os acordos de cooperação se concentram especialmente nas parcerias para a região das Américas com os Estados Unidos da América, Colômbia, Chile e Argentina, e, para a Europa, Portugal, França, Espanha e Itália, que continuam sendo os países de maior interesse para a Comunidade Acadêmica. No Continente Asiático, destaca-se a cooperação com a China e o Japão. Os acordos firmados com o Continente Africano abrangem Moçambique, África do Sul, Argélia e Camarões. Na Oceania, a Austrália e a Nova Zelândia são parceiras privilegiadas (UnB, 2017b).

\subsection{Plano de Internacionalização}

Desde 2018, a UnB conta com um plano de Internacionalização que definiu a linhas-mestras a serem seguidas para a consolidação do processo de internacionalização da UnB.

O Plano de Internacionalização 2018-2022 foi aprovado por unanimidade na 579a reunião do Conselho de Ensino, Pesquisa e Extensão (CEPE), no dia 26 de abril de 2018. É o primeiro documento da instituição sobre o assunto em 56 anos. O texto de 40 páginas descreve o panorama atual da Universidade, traçando diretrizes, objetivos e prazos que deverão ser cumpridos nos próximos quatro anos. A finalidade principal é garantir perenidade às ações desenvolvidas, nesse sentido, dentro da UnB. (RABELO, 2018)

\subsection{Internacionalização em Casa}

Embora a mobilidade acadêmica internacional seja a forma mais comum de internacionalização do ensino superior, a chamada "internacionalização em casa", que trata da internacionalização no âmbito interno das IES e dos países, tende a se consolidar no Brasil de diversas formas como, por exemplo, por meio da internacionalização dos seus currículos e da presença de professores e estudantes internacionais. A "internacionalização em casa" promove uma aceitação dos valores de outros povos, outras culturas, religiões e costumes, e proporciona a busca de soluções novas para problemas novos ou pouco familiares (TEIXEIRA, 2017). 
Uma das principais ações é a celebração de acordos de cooperação, que gera efeitos de universalização tais como o trabalho em rede, o intercâmbio mútuo de lições aprendidas, a complementaridade de ações acadêmicas de pesquisa e formação, maior valor agregado maior legitimidade social e maior visibilidade, entre outros (UnB, 2017b).

\subsection{Intercâmbio por meio de Acordos}

Os acordos bilaterais proporcionam ações de mobilidade acadêmica de professores, estudantes e técnicos, para dentro e para fora da instituição. A INT recebe, orienta e acompanha os alunos internacionais desses acordos. Também organiza a mobilidade de seus alunos para os mais diversos países e instituições. Seleciona os alunos da graduação para participar dos acordos como, por exemplo, o do Supremo Tribunal Federal, da Capes/Fipse, Capes/Brafitec, entre outros (UnB, 2017b).

\subsection{Alunos Internacionais na UnB}

Atualmente, a UnB está atendendo estudantes internacionais de diversos países. Em 2017, contava com 302 estudantes de pós-graduação e 210 estudantes de graduação, por meio de acordos bilaterais ou multilaterais (UnB, 2018a).

Ocorreu um significativo aumento entre o número de alunos enviados para outros países e o número de alunos recebidos de outros países pela Universidade de Brasília. Parte desse aumento deveu-se ao Programa Ciências sem Fronteiras, do Ministério da Educação, que contemplou um número bem elevado de alunos da $\mathrm{UnB}$, enquanto os alunos recebidos chegam à UnB por meio do Programa de Estudantes Convênio-Graduação Programa de Estudantes Convênio-Graduação (PEC-G) e do Programa de Estudantes Convênio-Pós-Graduação (PEC-PG), que são programas especiais criados pelo governo federal (Decreto Presidencial n. 7.948), como uma parceria entre o Ministério das Relações Exteriores (MRE), o Ministério da Educação e as Instituições de Ensino Superior do país (UnB, 2017a).

O Programa MARCA foi desenvolvido e implementado pelo Setor Educacional do MERCOSUL para promover a excelência acadêmica por meio de sistemas de avaliação e a acreditação e mobilidade de alunos, docentes e pesquisadores entre instituições de ensino superior de países da região. É coordenado por um acordo de cooperação educacional entre os países membros. No Brasil, a Coordenação de Aperfeiçoamento de Pessoal de Nível Superior (Capes) e a Secretaria de Educação Superior (SESu) do MEC são os responsáveis por esse acordo (UnB, 2017b).

De tal forma, este estudo pretende descrever o atual processo de internacionalização da UnB e poderá servir para estruturar, orientar e dar continuidade às novas ações de internacionalização a serem implementadas segundo o Plano de Internacionalização da UnB para o período 2018-2022.

\section{Metodologia}

A abordagem metodológica adotada neste estudo tem como referência a pesquisa qualitativa e quantitativa, exploratória com abordagem indutiva, com a intenção de se apresentar uma descrição analítica das atividades conduzidas pela INT/UnB, pois, desde abril de 2018, 
esta Instituição de Educação Superior (IES) conta com um Plano de Internacionalização com diretrizes até então não sistematizadas. O procedimento metodológico foi a pesquisa bibliográfica, com estudo de caso com método qualitativo.

A pesquisa bibliográfica foi essencial para contextualizar o tema do ponto de vista teórico, por trazer a definição da internacionalização das IES.

A consulta a fontes primárias, nos registros oficiais da UnB e da INT, sobre sua história e evolução, bem como os dados quantitativos sobre a evolução dos números e percentuais de acordos internacionais, por país, realizados pela UnB, por meio da INT, dá sustentação aos resultados alcançados, a conclusão e norteará os desafios ainda colocados à internacionalização da UnB.

A metodologia adotada na pesquisa foi dividida em duas partes: na promeira parte foi feita uma revisão bibliográfica de obras e de artigos científicos de pesquisadores do Brasil e do exterior, realizada no Repositório de Periódicos Capes. As palavras-chave para a busca foram: internacionalização, educação superior, inovação, com a pretensão de se obter respostas que apontam os desafios à internacionalização das instituições de ensino superior. Na segunda parte foi realizada uma análise dos documentos oficiais da UnB disponibilizados ao público pelo Decanato de Planejamento (DPO), bem como pelo portal da UnB.

\section{Resultados e Discussão}

A partir da avaliação dos documentos pesquisados nas bases de dados disponíveis no Decanato de Planejamento da Universidade de Brasília, verificou-se que a UnB contava em 2017, com 42.421 estudantes, sendo 7.724 estudantes de pós-graduação e 34.697 estudantes de graduação, com 2.404 professores e 3.024 servidores técnico-administrativos para ofertar 155 cursos de graduação e 154 programas de pós-graduação, em 20 centros de pesquisa (UnB, 2017a).

Em seus relatórios anuais, a UnB (2017b) detectou um sério obstáculo a ser enfrentado pela UnB para a efetiva consolidação de seu perfil internacional - a oferta restrita de disciplinas em inglês ou em outras línguas estrangeiras na maioria dos cursos de graduação e pós-graduação. Essa situação reduz de forma substantiva o número e a variedade de estudantes estrangeiros que poderiam realizar programas de intercâmbio na UnB. Também inibe a celebração de um maior número de acordo de cooperação bilaterais e coloca em discussão o princípio da reciprocidade celebrado nos acordos.

A oferta de cursos em língua inglesa ou em espanhol proporcionaria a atualização e o aprimoramento para os professores da UnB nestas duas línguas estrangeiras, e ampliaria o número de publicações científicas de docentes em inglês ou espanhol em revistas e livros de editoras internacionais. O que também mitigaria as dificuldades de divulgação científicas efetivas ocasionadas pela falta de compartilhamento das informações internas e externas, entre outras, às quais a INT está atenta e buscando as melhores soluções.

A UnB está aberta para receber alunos internacionais nas seguintes modalidades: 1) Aluno de intercâmbio por meio de convênio; 2) Estudante de Graduação Visitante; 3) Pesquisador Colaborador Júnior de Pós-Graduação; 4) Programa Cortes Supremas MERCOSUL para estudantes de Direito (Graduação e Pós-Graduação) do MERCOSUL para a realização de estágio 
no Supremo Tribunal Federal; 5) Programa de Intercâmbio MARCA: Programa de Mobilidade Acadêmica Regional para Cursos de Graduação nos países signatários e convidados (Brasil, Argentina, Uruguai, Paraguai, Chile, Bolívia, Colômbia e Venezuela) nos cursos de Arquitetura e Agronomia. Os alunos devem estar regularmente matriculados em uma das instituições conveniadas à Universidade de Brasília (UnB, 2018a).

\subsection{Alunos Internacionais na UnB em 2017}

Em 2017, a UnB atendeu alunos internacionais de diversos países, sendo 302 estudantes de pós-graduação e 210 estudantes de graduação, por meio de acordos bilaterais ou multilaterais. Apesar de muito esforço institucional, há um desequilíbrio entre envio e recebimento de alunos no intercâmbio institucional da UnB. Os fatores desse desequilíbrio ainda necessitam de estudos para estabelecer os principais obstáculos para, então, corrigir (UnB, 2018a).

Os estudantes da Universidade de Brasília têm uma preferência para realizar pesquisas e/ ou estudos em Centros Europeus, seguido dos países da América. Por outro lado, a África, a Ásia e a Oceania receberam 44, 44 e sete estudantes, respectivamente, no período de 2008 a 2016 (UnB, 2017b).

O mesmo acontece com seus estudantes, que, tanto na graduação como na pós-graduação, estabelecem vínculos acadêmicos e de pesquisa com estudantes de outros países. Nos campi da UnB, nota-se a cada dia um número maior de estudantes estrangeiros que aprofundam suas atividades acadêmicas colaborativas, e ampliam a diversidade cultural, científica e tecnológica na Universidade. Os acordos com parceiros acadêmicos internacionais representam uma demanda crescente à qual a UnB responde prontamente.

Entretanto, verifica-se a necessidade de um projeto de "internacionalização em casa" que contemple os novos campi da UnB, pois a internacionalização não pode ficar restrita ao campus Darcy Ribeiro, e deve ser desenvolvida a cultura da internacionalização em toda a Instituição. Com o Plano de Internacionalização (2018-2022) esse cenário tende a mudar (UnB, 2018b).

\subsection{Matrícula Cortesia}

Com base no Decreto n. 89.758, de 6 de junho de 1984, a UnB oferece Matrícula Cortesia, que é uma forma de ingresso de aluno oriundo de país que assegure o regime de reciprocidade com o Brasil, independentemente da existência de vaga e com isenção do vestibular ou outro tipo de seleção. Destina-se a funcionário estrangeiro de missão diplomática ou repartição consular de carreira no Brasil e seus dependentes legais; funcionário ou técnico estrangeiro de organismo internacional que goze de privilégios e imunidades em virtude de acordo entre o Brasil e a sua organização, assim como seus dependentes legais; bem como a técnico estrangeiro que preste serviço em território nacional, no âmbito de acordo de cooperação técnica ou cultural firmado entre o Brasil e seu país de origem, assim como seus dependentes legais (UnB, 2018b). 


\subsection{Português para Estrangeiros}

Ações de internacionalização também se verificam na oferta de cursos de Português para Estrangeiros a candidatos selecionados para o Programa Estudante-Convênio de Graduação (PEC-G), conforme acordos internacionais firmados entre o Ministério da Educação (MEC) e o Ministério das Relações Exteriores (MRE), operacionalizados pelo Núcleo de Ensino e Pesquisa em Português para Estrangeiros (NEPPE), o qual oferece cursos de português aos intercambistas e à comunidade estrangeira pertencente ou não ao corpo diplomático, pois seu objetivo é coordenar, supervisionar e promover o ensino de cursos de Português para Estrangeiros, incluindo cursos regulares bimestrais e intensivos nos períodos de recesso, além de fortalecer e incentivar a pesquisa científica na produção de conhecimentos na área de Português para Estrangeiros (UnB, 2017a).

\subsection{A UnB nas Redes Universitárias}

As redes universitárias são órgãos que reúnem representantes de universidades com objetivos comuns. As redes globais de universidade não estão apenas proliferando entre as instituições, elas também cruzam setores para engajar novos parceiros e alavancar recursos de parcerias a fim de beneficiar todos os parceiros e criar estratégias regionais de fortalecimento acadêmico (UnB, 2017a).

Como Redes, as Instituições se juntam e cooperam por meio de múltiplos temas para a troca de informações e boas práticas, estabelecem um benchmark de suas atividades, criam novos conhecimentos com pesquisa e programas conjuntos de formação, facilitam a mobilidade de docentes e estudantes, aperfeiçoam recursos, aumentam a capacidade, promovem $e$ defendem serviços e valores. A gestão de relacionamentos de maneira respeitosa e produtiva em todas as fronteiras internacionais é o principal desafio de competência na manutenção das redes globais (UnB, 2017a).

A Universidade de Brasília faz parte de várias redes, internacionais e nacionais, como: a Organização Universitária Interamericana (OUI), o Grupo Coimbra, Asociación de Universidades Grupo Montevideo (AUGM), a Associação das Universidades de Língua Portuguesa (AULP), a Associação das Universidades Amazônicas, a Associação Brasileira dos Reitores das Universidades Estaduais e Municipais (Abruem), o Conselho de Reitores das Universidades Brasileiras (Crub), a Associação Nacional dos Dirigentes das Instituições Federais de Ensino Superior (Andifes), etc. (UnB, 2018b). A Organização Universitária Interamericana, com mais de 30 anos de experiência, acompanha as tendências no campo da cooperação interamericana entre as Instituições Ensino Superior, e fortalece a criação de espaços comuns de ensino superior, considerando as necessidades de seus membros, na formação da geração do futuro. Atualmente, a OUI conta com 321 membros que incluem as instituições de ensino superior e associações universitárias, de 26 países das Américas, divididos em nove regiões: América Central, Brasil, Canadá, Caribe, Colômbia, Cone Sul, México, Estados Unidos e Países Andinos (UnB, 2017a).

O Grupo Coimbra (GCUB) de Universidades Brasileiras é uma rede de universidades nacionais - federal, estadual, confessional e comunitário - que promove a integração interinstitucional e internacional, mediante programas de mobilidade docente e discente, para o processo de internacionalização da rede universitária nacional com suas parcerias internacionais. Por 
meio da Universidade de Coimbra, a mais antiga do mundo lusófono, o Grupo integra-se às universidades do mundo ocidental, reunidas no Grupo Coimbra de Universidades Europeias, com as quais mantém vínculos de intercâmbio científico, pedagógico e cultural (UnB, 2017a).

A Associação das Universidades de Língua Portuguesa (AULP) é um organismo internacional que promove a cooperação e a transferência de informações entre Universidades e Institutos Superiores. Conta com 140 membros dos oito países de língua oficial portuguesa - Angola, Brasil, Cabo Verde, Guiné-Bissau, Moçambique, Portugal, São Tomé e Príncipe, Timor Leste e Macau. Sua missão é facilitar a comunicação entre os membros da AULP para desenvolver o ensino da língua portuguesa no mundo e estimular a pesquisa e o intercâmbio dos docentes e discentes. Atua no reforço das relações desta Associação com a Comunidade de Países de Língua Portuguesa (CPLP), da qual é Observador Consultivo, e pode intervir em áreas temáticas e assumir a função de assessoria científica por solicitação da CPLP (UnB, 2017a).

Casa da Cultura a América Latina (CAL) da UnB, Criada em 1987, para promover e divulgar a arte e a cultura latino-americana, a Casa da Cultura da América Latina (CAL) se consolidou como um espaço voltado para a promoção das culturas ibero, latina e africana, em todas as suas vertentes e linguagens. Além da promoção de eventos, é um espaço de estudo e de preservação do patrimônio cultural e artístico da UnB. Possui um importante acervo de arte moderna, contemporânea, popular e etnográfica. O Cinema da Casa da Cultura da América Latina (CineCAL) exibe filmes produzidos, em sua maioria, nos países da América Latina, África e Península Ibérica. Oficinas de arte, espetáculos teatrais, cursos, palestras, seminários, entre outros, também fazem parte da programação da Casa (UnB, 2017b).

\subsection{Instituto Confúcio (IC) na UnB}

O Instituto Confúcio (IC) é resultado de Acordo de Cooperação firmado entre a Matriz do Instituto Confúcio da China e a Fundação Universidade de Brasília. O IC da UnB está sob a gestão do Departamento de Línguas Estrangeiras e Tradução do Instituto de Letras da UnB e desenvolve atividades de ensino, pesquisa e extensão. O IC da UnB conta com uma biblioteca com mais de 5.000 exemplares doados pela sede do Instituto Confúcio da China. De 2008 a 2018, já atendeu a 824 alunos, dos quais, 428 são estudantes da comunidade externa e 396 estudantes, professores ou servidores da UnB (UnB, 2017b).

\section{Considerações Finais}

A Universidade de Brasília $(\mathrm{UnB})$ é resultado de pensamento e ação voltados tanto para dentro do País quanto para fora deste. Sua localização geográfica privilegiada, perto dos centros decisórios de poder nacional, das representações do corpo diplomático acreditado junto ao governo brasileiro, dos organismos internacionais, e também das agências de fomento e outros parceiros indispensáveis à sua evolução, como o Ministério da Educação (MEC), a Coordenação de Aperfeiçoamento de Pessoal de Nível Superior (Capes), o Conselho Nacional de Desenvolvimento Científico e Tecnológico (CNPq), da Empresa Brasileira de Pesquisa Agropecuária (Embrapa), SEBRAE nacional e do DF, etc., potencializa sua vocação para se tornar um grande polo acadêmico internacional do Centro-Oeste do Brasil. 
Ecoando à definição sugerida por De Wit (2015), a UnB, fortemente inserida na comunidade global, consolida parcerias com as mais diversas instituições de educação superior no Brasil e no mundo. Ela se acomoda nas mais diversas definições de uma universidade internacionalizada, pois a maioria de seus professores/pesquisadores complementou, ou ainda complementa suas formações acadêmicas e de pesquisas em outros países por meio de parcerias estratégicas para o desenvolvimento não apenas da Universidade, mas do Brasil como um todo. A INT dispobiliza em sua página eletrônica (www.int.unb.br) a situação atual de todos os acordos em vigência.

Por outro lado, os acordos bilaterais da UnB e seus parceiros proporcionaram a mobilidade de estudantes da UnB para todos os continentes, e a sua prevalência de destino para o Continente Europeu (UnB, 2017b).

Entre 2008 e 2018, a UnB está trilhando o caminho da internacionalização a passos largos, pois sua comunidade acadêmica é bem exigente e bem formada dentro e fora do país. Com a expansão que ocorreu nos últimos anos na UnB verificou-se a necessidade de um plano de internacionalização que leve em consideração muitos dos aspectos que podem consolidar sua vocação internacional.

A UnB segue atuante em todas as áreas do conhecimento, aberta às principais demandas do Brasil e do mundo, com o olhar apontado para o futuro e, estima-se pelo caminho já trilhado, que a Universidade se consolida como organismo indispensável ao desenvolvimento da sociedade.

Ao ultrapassar fronteiras e divulgar o conhecimento adquirido nas mais diversas instituições estrangeiras de ensino superior e de pesquisa, a UnB busca a excelência e a inovação acadêmica para ultrapassar seus próprios limites cotidianamente. A Internacionalização de uma instituição de ensino superior é um processo, e, como tal, está em constante transformação. Na UnB assim acontece.

\section{Referências}

BRASIL. Programa de Internacionalização. Brasília, DF: MEC, [2018]. Disponível em: http://www. capes.gov.br/images/stories/download/editais/10112017-Edital-41-2017-Internacionalizacao-PrInt-2. pdf. Acesso em: 12 jan. 2018.

DE WIT, H. Quality Assurance and Internationalization. Trends, challenges and opportunities. INQAAHE conference, Chicago, U.S., 2015.

INEP - INSTITUTO NACIONAL DE ESTUDOS E PESQUISAS EDUCACIONAIS ANÍSIO TEIXEIRA. Dados do censo da educação superior: as universidades brasileiras representam $8 \%$ da rede, mas concentram 53\% das matrículas. Em 3 de outubro de 2018. Disponível em: http://portal.inep. gov.br/artigo/-/asset_publisher/B4AQV9zFY7Bv/content/dados-do-censo-da-educacao-superior-asuniversidades-brasileiras-representam-8-da-rede-mas-concentram-53-das-matriculas/21206. Acesso em: 13 de out. 2018.

KNIGHT, J. Updating the Definition of Internationalization, International Higher Education. Boston: Issue 33, 2003. Disponível em: https:/www.bc.edu/content/dam/files/research_sites/cihe/pdf/ IHEpdfs/ihe33.pdf. Acesso em: 7 mar. 2020.

MORHY, L. et al. Universidade em Questão. Brasília, DF: Editora Universidade de Brasília, 2003. v. 1. 
MORHY, L. et al. Universidade no mundo: Universidade em Questão. Brasília, DF: Editora Universidade de Brasília, 2004.

RABELO, N. Plano de Internacionalização da UnB. Publicado em: 20 jun. 2018. Disponível em: http://www.int.unb.br/br/institucional/plano-de-internacionalizacao. Acesso em: 21 jun. 2018.

TEIXEIRA, E. A Internacionalização das universidades: tendências e desafios, 2017. Grupo Coimbra. Disponível em: http://www.grupocoimbra.org.br/. Acesso em: 22 mar. 2018.

UnB - UNIVERSIDADE DE BRASÍLIA. Anuário Estatístico da UnB 2016. Período: 2011 a 2015). Decanato de Planejamento e Orçamento - DPO. Brasília, DF: Universidade de Brasília, 2017a.

UnB - UNIVERSIDADE DE BRASÍLIA. Estatuto e Regimento 2011. Disponível em: http://www. unb.br/images/Noticias/2016/Documentos/regimento_estatuto_unb.pdf. Acesso em: 29 jun. 2018.

UnB - UNIVERSIDADE DE BRASÍLIA. Assessoria de Assuntos Internacionais, INT. Relatório de Avaliação da INT - 2017. Decanato de Planejamento, Orçamento e Avaliação Institucional - DPO, Brasília, DF, 2017b

UnB - UNIVERSIDADE DE BRASÍLIA. Assessoria de Assuntos Internacionais, INT. Relatório de Gestão da INT - 2018. Decanato de Planejamento, Orçamento e Avaliação Institucional - DPO. UnB, Brasília-DF, 2018a.

UnB - UNIVERSIDADE DE BRASÍLIA. Assessoria de Assuntos Internacionais, INT. Plano de Internacionalização da UnB. Publicado em: 12 maio 2018b. Disponível em: http://www.int.unb.br/br/ institucional/plano-de-internacionalizacao. Acesso em: 21 jun. 2019.

\section{Sobre as Autoras}

\section{Regina Coeli Andrade Marques}

E-mail: reginamarqueseditora@gmail.com

Graduação em Licenciatura em Física pela Universidade de Brasília (UnB).

Endereço profissional: Universidade de Brasília, Campus Universitário Darcy Ribeiro, Edifício Reitoria, Sala BT 1/7, Brasília, DF. CEP: 70910-900.

\section{Maria Hosana Conceição}

E-mail: hosanac@unb.br

Doutora em Química pela Universidade de Brasília (UnB)

Endereço profissional: Universidade de Brasília, Campus Universitário Darcy Ribeiro, Edifício CDT, Brasília, DF. CEP: 70904-970. 